\title{
The T, $p$-Dependence of the Chemical Shift of the Hydroxyl Protons in Deeply Supercooled Methanol and Water
}

\author{
Liuping Chen, Thomas Gross ${ }^{a}$, and Hans-Dietrich Lüdemann ${ }^{a}$ \\ Institute of Physical Chemistry, School of Chemistry and Chemical Engineering, \\ Zhongshan (Sun Yat-Sen) University, Guangzhou 510275, People's Republic of China \\ a University of Regensburg, Institute of Biophysics and Physical Biochemistry, D-93040 Regensburg \\ Reprint requests to Prof. H.-D. L.; Fax: 0941-943-2479; \\ E-mail: Hans-Dietrich.Luedemann@Biologie.Uni-Regensburg.DE
}

Z. Naturforsch. 55a, 473-477 (2000); received November 11, 1999

The hydroxyl proton chemical shifts $\delta(\underline{\mathrm{H}}-\mathrm{O})$ of supercooled methanol $\left(T_{\min }=149 \mathrm{~K}\right)$ and water have been determined $\left(T_{\min }=183 \mathrm{~K}\right)$, and the pressure dependence of these shifts was measured up to $200 \mathrm{MPa}$. In both compounds the downfield shift of $\delta(\underline{\mathrm{H}}-\mathrm{O})$ continues down to the lowest temperatures reached. This result disagrees with the two state models for the hydrogen bond formation in both liquids. The isotherms $\delta\left(\underline{\mathrm{H}}_{2} \mathrm{O}\right)$ show for $T \leq 273 \mathrm{~K}$ an upfield shift that becomes more pronounced with decreasing temperature. For $\delta\left(\underline{\mathrm{H}}-\mathrm{O}-\mathrm{CH}_{3}\right)$, increasing $p$ causes at all temperatures a downshift.

Key words: NMR; Supercooling; Pressure; Methanol; Water.

\section{Introduction}

The chemical shift of hydroxyl- and amino protons is a very sensitive marker for the formation of hydrogen bonds [1]. The chemical shift of a given proton involved in this type of bonding depends in a complex manner upon both bond lengths of the $-\mathrm{O}-\mathrm{H} \cdots \mathrm{O}$-fragment, on the deviation from linearity of this partial structure, on the three other substituents of the two oxygens, and in the condensed states of matter also on the influence that the molecules in the immediate vicinity have upon the electron density at the site of the proton [2].

It is thus not surprising that a comprehensive quantitative theoretical model for the $T$, $p$-dependence of the chemical shift is still missing although compounds like ethylene, glycol and methanol [3] have been used for decades as NMR-thermometers by calibrating their hydroxyl proton chemical shift as function of temperature.

Recently Dougherty [4] showed that the lengths and strengths of hydrogen bonds depend sensitively on $T$ and $p$, thus showing that all attempts to fit the observable changes of chemical shift in equilibria involving hydrogen bonds by two state models involving one defined shift each for the bonded and the nonbonded hydroxyl protons are at best first order approximations. A more realistic description of hydrogen bonding in water and the alcohols should apply continuum models.

During the last years new reliable proton NMR spectra of methanol [5] and water [6, 7] at temperatures around and above the respective critical temperatures be- came available, establishing that at sufficiently high temperatures and low densities the chemical shift of the hydroxyl protons is constant in a wide range of $T$ and $p$.

These results prompted us to extend the measurements of the proton chemical shift of water and methanol into the metastable supercooled region in order to learn whether also in this range the chemical shift becomes less sensitive to changes in $T$ and $p$. These measurements extend into the deeply supercooled range for both compounds and should help in clarifying the recent discussions about the metastable phases of water [8].

\section{Experimental}

Substances: Ultrapure water was freshly drawn from a Milli-Q unit. To the bulk water samples $0.5 \%$ of 3-(trimethylsilyl) propionic acid sodium salt (TPS) (Uvasol, Merck, Darmstadt, FRG) was added as an internal reference. For the extreme ranges of supercooling, water in oil emulsions had to be used. The oil consisted of $30 \% \mathrm{w} / \mathrm{w}$ methylcyclopentane, $30 \% \mathrm{w} / \mathrm{w}$ methylcyclohexane, and $40 \% \mathrm{w} / \mathrm{w}$ hexamethyldisiloxane (HMDS), the latter being used as an external reference. Methanol (Uvasol, Merck, Darmstadt, FRG) was taken from a freshly opened bottle and used without further purification. The chemical shift of the methanol hydroxyl is referenced to the methylprotons.

The spectra were taken in strengthened glass capillaries of the Yamada type [9]. For the emulsions, capillar- 
ies with i.d. $1.2 \mathrm{~mm}$ and o.d. $5 \mathrm{~mm}$ were used; details of the design have been published [10]. The bulk samples of water and all methanol samples were measured in very thin capillaries (i.d. $0.1 \mathrm{~mm}$, o.d. $1.2 \mathrm{~mm}$ ) [11].

A Bruker MSL 300 spectrometer, working at a proton frequency of $300 \mathrm{MHz}$, in combination with a home built probe head was used. The samples were thermostated with a flow of nitrogen. Temperatures are controlled to $\pm 1 \mathrm{~K}$ with a metal sheathed chromel/alumel thermocouple. The whole high pressure apparatus is described in [10].

\section{Results and Discussion}

The chemical shift of the hydroxylproton $\delta(\mathrm{OH})$ in methanol has since the very early studies [3] been measured relative to the methylprotons $\delta\left(\mathrm{CH}_{3}\right)$. All recent publications $[5,12,13]$ of the $T, p$-dependence of this shift difference agree with the present work within the limits of experimental error. Our work extends the high pressure data into the low temperature range $150 \mathrm{~K}<T<$ $280 \mathrm{~K}$.

Figure 1 collects some isotherms of $\delta(\mathrm{OH})-\delta\left(\mathrm{CH}_{3}\right)$. The ambient pressure data by Van Geet [3] ended at $175 \mathrm{~K}$ and are slightly extended by our data. At all temperatures this quantity increases with pressure. This is taken as an indication for shifting the hydrogen bond equilibrium with pressure in the direction of more and/or stronger hydrogen bonds. The slope of these isotherms becomes smaller with decreasing temperature which is easily explained by the reduced compressibility of the cold liquid. In Fig. 2 the $20 \mathrm{MPa}$ isobar for $\delta(\mathrm{OH})-$ $\delta\left(\mathrm{CH}_{3}\right)$ is given. At high temperatures and therefore low density the $T$-dependence of this quantity is very weak, indicating the predominance of free nonbonded hydrox$\mathrm{yl}$ groups. Already at temperatures around $600 \mathrm{~K}$ the chemical shift starts to change rapidly with pressure and with some good will the slope of the curve could be interpreted as the onset of a sigmoidal $T$-dependence of $\delta$ $(\mathrm{OH})-\delta\left(\mathrm{CH}_{3}\right)$. However, this "sigmoidal curve" does not flatten out at further lowering of $T$. This is already quite clearly seen in the data by Van Geet [3]. It is in our opinion a clear indication that the description of the hydrogen bonding in a two states model with constant $\delta$ $(\mathrm{OH})_{\text {free }}$ and $\delta(\mathrm{OH})_{\text {bonded }}$ is not fully adequate. One can however attempt to describe the region between $250 \mathrm{~K}$ and $600 \mathrm{~K}$, where the change with temperature are most pronounced as the range where completely hydrogen bonded associates are formed [5]. The smaller increase

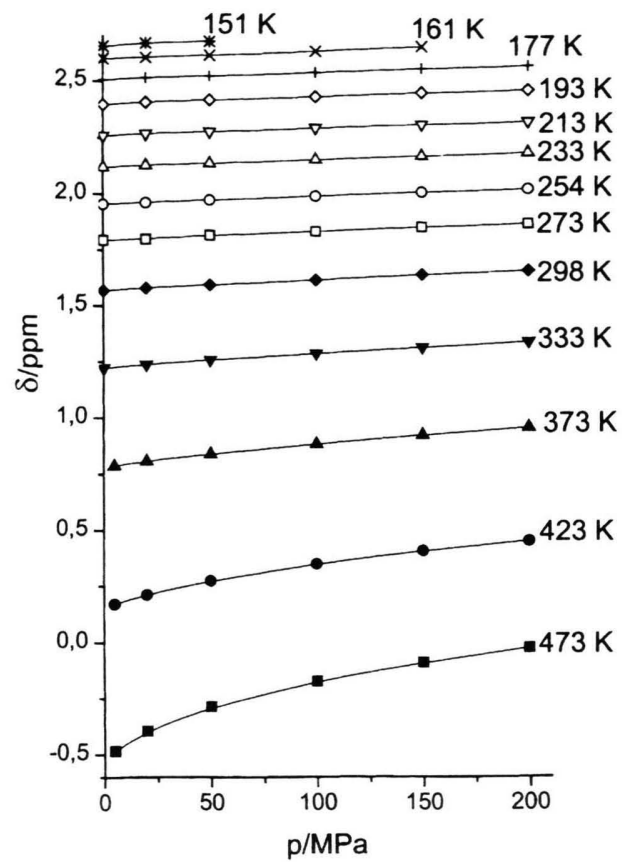

Fig. 1. Isotherms of the proton chemical shift of the hydroxyl protons in neat methanol. Referenced to the methyl protons. $\delta=\delta\left(\underline{\mathrm{H}}-\mathrm{OCH}_{3}\right)-\delta\left(\mathrm{C}_{3}-\mathrm{OH}\right)$.

Table 1. Chemical shift of the hydroxyl proton in neat methanol $\delta(\underline{H}-\mathrm{O})-\delta\left(\mathrm{CH}_{3}\right)$.

\begin{tabular}{|c|c|c|c|c|c|c|c|}
\hline \multirow[t]{2}{*}{$T / \mathrm{K}$} & \multicolumn{7}{|c|}{$p / \mathrm{MPa}$} \\
\hline & 0.1 & 5 & 20 & 50 & 100 & 150 & 200 \\
\hline 73 & & -0.484 & -0.396 & -0.289 & -0.176 & -0.093 & -0.027 \\
\hline 23 & & 0.169 & 0.211 & 0.272 & 0.347 & 0.405 & 0.449 \\
\hline 373 & & 0.787 & 0.806 & 0.838 & 0.882 & 0.921 & 0.954 \\
\hline 333 & 1.221 & & 1.235 & 1.256 & 1.285 & 1.310 & 1.336 \\
\hline 98 & 1.567 & & 1.577 & 1.590 & 1.613 & 1.635 & 1.655 \\
\hline 73 & 1.794 & & 1.797 & 1.814 & 1.831 & 1.849 & 1.867 \\
\hline 254 & 1.955 & & 1.960 & 1.972 & 1.990 & 2.006 & 2.023 \\
\hline 233 & 2.120 & & 2.127 & 2.135 & 2.153 & 2.168 & 2.186 \\
\hline 213 & 2.259 & & 2.266 & 2.276 & 2.293 & 2.307 & 2.324 \\
\hline 193 & 2.396 & & 2.405 & 2.416 & 2.431 & 2.448 & 2.465 \\
\hline 177 & 2.506 & & 2.514 & 2.522 & 2.539 & 2.556 & 2.572 \\
\hline 16 & 2.599 & & 2.603 & 2.613 & 2.632 & 2.653 & \\
\hline 15 & 2.654 & & 2.666 & 2.674 & & & \\
\hline 1 & 2.663 & & & & & & \\
\hline
\end{tabular}

observed for $T \leq 250 \mathrm{~K}$ may be mainly due to the optimisation of the energies and geometries of the hydrogen bonds demanded by recent theories [4] as a result of the removal of thermal energy. The methanol molecules can participate in hydrogen bonding with one donor and two acceptor sites. Thus only twodimensional aggregates, chains, branched chains and rings may form upon asso- 


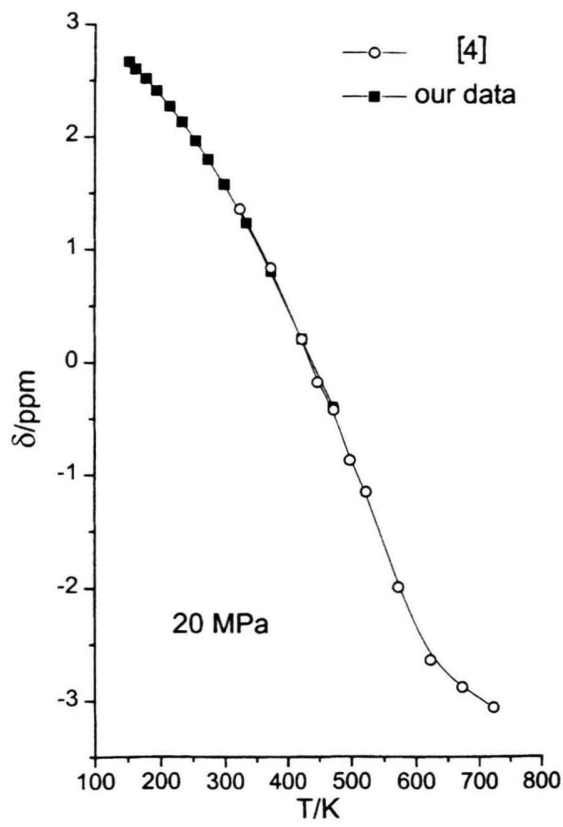

Fig. 2. $20 \mathrm{MPa}$ isobar of the proton chemical shift of the hydroxyl protons in neat methanol $\delta=\delta\left(\underline{\mathrm{H}}-\mathrm{OCH}_{3}\right)-\delta\left(\mathrm{CH}_{3}-\mathrm{OH}\right)$. Our data overlap for $T>175 \mathrm{~K}$ with the older results by Van Geet [3].

ciation. It is obvious from inspection of Fig. 2 that the $T$ dependence of the chemical shift cannot be described in the most simple two state model where the hydrogen bond formation is described by association constants that are independent of chain lengths, and the concentration of monomers and associated molecules is derived by assigning one $T, p$ independent chemical shift each to the free and the bonded hydroxyl protons [14]. However all more complicated models of association involve too many freely adjustable parameter, that are impossible to derive from the NMR data alone.

\section{Water}

The chemical shift data reported for methanol were all taken with the same internal reference: the methylprotons of the alcohol itself. Most of the data published about the proton chemical shift of water under extreme conditions $[6,7,15-17]$ were not taken with the established internal temperature-pressure-standard (TPS) but used several different inert molecules and a mixture of internal and external standards. In order to compare and correlate all data sets, we therefore began a series of measurements with bulk water in capillaries of $0.1 \mathrm{~mm}$ i.d. using an addition of $0.5 \% \mathrm{w} / \mathrm{w}$ TPS as an internal standard.
Table 2. Proton chemical shift of water in emulsion. Referenced to external hexamethyldisiloxane.

\begin{tabular}{lllllll}
\hline$T / \mathrm{K}$ & \multicolumn{2}{l}{$p / \mathrm{MPa}$} & \multicolumn{5}{l}{} \\
\cline { 2 - 7 } & 1 & 20 & 40 & 100 & 150 & 200 \\
\hline 298 & 4.703 & 4.693 & 4.690 & 4.685 & 4.679 & 4.680 \\
284 & 4.862 & 4.861 & 4.856 & 4.836 & 4.820 & 4.801 \\
273 & 4.978 & 4.958 & 4.941 & 4.915 & 4.890 & 4.867 \\
264 & 5.088 & 5.058 & 5.036 & 5.001 & 4.987 & 4.983 \\
253 & 5.212 & 5.188 & 5.167 & 5.116 & 5.084 & 5.067 \\
244 & 5.335 & 5.270 & 5.251 & 5.203 & 5.180 & 5.154 \\
233 & 5.575 & 5.499 & 5.403 & 5.324 & 5.283 & 5.244 \\
223 & & & & 5.394 & 5.350 & 5.331 \\
213 & & & & & & 5.418 \\
203 & & & & & & 5.509 \\
192 & & & & & & 5.615 \\
188 & & & & & & 5.638 \\
183 & & & & & & \\
\hline
\end{tabular}

Table 3. Proton chemical shift of water referenced to internal TPS.

\begin{tabular}{|c|c|c|c|c|c|c|}
\hline \multirow[t]{2}{*}{$T / \mathrm{K}$} & \multicolumn{6}{|c|}{$p / \mathrm{MPa}$} \\
\hline & 1 & 20 & 40 & 100 & 150 & 200 \\
\hline 423 & 3.758 & 3.773 & 3.792 & 3.838 & 3.873 & 3.904 \\
\hline 373 & 4.127 & 4.139 & 4.151 & 4.185 & 4.212 & 4.235 \\
\hline 354 & 4.290 & 4.299 & 4.310 & 4.339 & 4.360 & 4.383 \\
\hline 333 & 4.472 & 4.479 & 4.486 & 4.508 & 4.525 & 4.542 \\
\hline 314 & 4.653 & 4.658 & 4.662 & 4.677 & 4.691 & 4.702 \\
\hline 298 & 4.805 & 4.808 & 4.812 & 4.820 & 4.828 & 4.840 \\
\hline 288 & 4.913 & 4.912 & 4.915 & 4.918 & 4.925 & 4.931 \\
\hline 273 & 5.074 & 5.072 & 5.068 & 5.062 & 5.062 & 5.063 \\
\hline 258 & 5.262 & 5.252 & 5.242 & 5.222 & 5.212 & 5.210 \\
\hline 253 & 5.324 & 5.309 & 5.296 & 5.272 & 5.260 & 5.253 \\
\hline 247 & & & & 5.326 & 5.311 & 5.303 \\
\hline 243 & & & & 5.381 & 5.361 & 5.350 \\
\hline 238 & & & & & 5.404 & 5.387 \\
\hline 23 & & & & & & 5.455 \\
\hline & & & & & & 5.516 \\
\hline
\end{tabular}

This internal standard eliminates the need for susceptibility corrections, that always carry an amount of uncertainty. Although it could be argued that the chemical shift of the internal standard might be influenced by temperature and pressure induced changes in the hydration structure of the trimethylsilylgroup. In Table 3 all data collected for the bulk phase with TPS as an internal standard are compiled. Table 2 gives all uncorrected emulsion data with hexamethyldisiloxane as external standard.

In $[5,6]$ benzene was used as an internal standard. Our data with the TPS standard overlap at $423 \mathrm{~K}$. Taking the accepted proton chemical shift of benzene $\delta=7.45 \mathrm{ppm}$ and transferring the data of $[5,6]$ for $20 \mathrm{MPa}$ to the TPS scale yields $\delta(423 \mathrm{~K})=3.74 \mathrm{ppm}$, compared to our result 


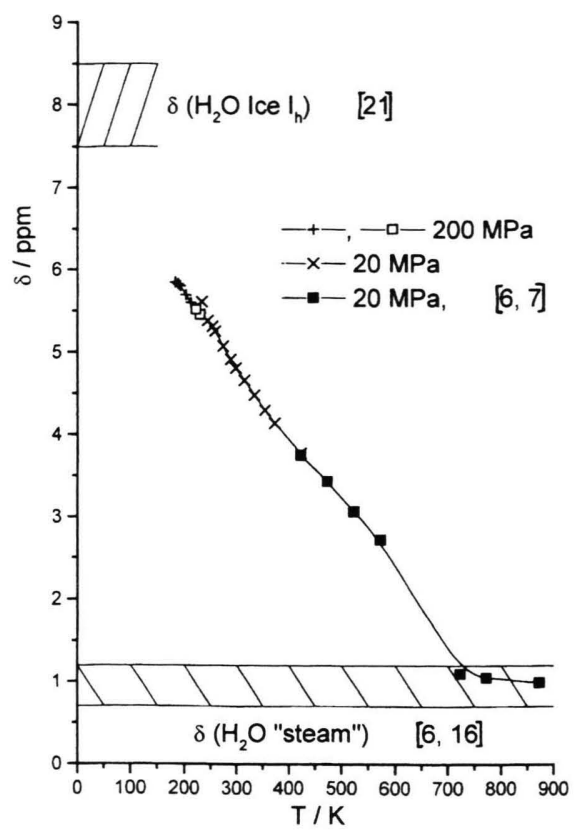

Fig. 3. Isobars of the proton chemical shift of water referenced to internal TPS $(0.5 \%)$. Previous data have been rescaled. For details see text. The shaded areas give the value for the proton chemical shift of low density steam (bottom) respectively ice $I_{\mathrm{h}}$.

of $\delta(423 \mathrm{~K})=3.77 \mathrm{ppm}$. Assuming that this rescaling is appropriate for the whole temperature range, we arrived at the data given in Figure 3. Hoffmann and Conradi [6] incorporated the data by Matubayasi et al. [7] into their compilation and state that these results agree with their data.

For $T>773 \mathrm{~K}$ and $p \leq 20 \mathrm{MPa}$ the chemical shift of the water protons shows only a very weak $T$, $p$-dependence $\delta\left(\mathrm{H}_{2} \mathrm{O}\right)$ on the TPS scale: $\delta$ varies between 0.75 and $0.95 \mathrm{ppm}$. This is to be compared to the Hindman [16] data of $0.69 \mathrm{ppm}$ measured between 403 and $463 \mathrm{~K}$ at pressures between 0.5 and $1.2 \mathrm{MPa}$. It is stated by both authors that this value of the chemical shift corresponds to the free nonbonded protons in water. In Fig. 3 the range indicated in the lower part gives the estimated uncertainty of this value. For $258 \mathrm{~K} \leq T \leq 373 \mathrm{~K}$, Hindman determined the chemical shift of the water protons at ambient pressure against methane as an external standard. Setting $\delta\left(\mathrm{CH}_{4}\right)=0.13 \mathrm{ppm}$ at the TPS scale yields very good agreement with our data, however the $T$-dependence of $\delta$ in our internally standardized sample is slightly smaller. In the temperature range given above this amounts to a divergence at the two scales by $\Delta \delta=0.05 \mathrm{ppm}$. This difference can either result from the uncertainties in the

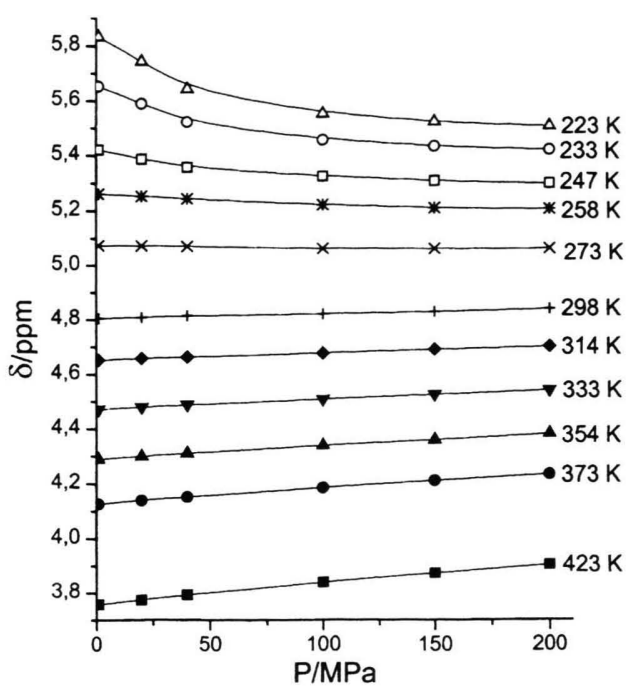

Fig. 4. Isotherms of the proton chemical shift of water referenced to internal TPS $(0.5 \%)$. The chemical shifts for the isotherms for $T \leq 253$ were calculated from the emulsion data.

susceptibility correction by Hindman [16], or indicate that TPS is not really a fully inert standard in water. Angell et al. [17] extended the ambient pressure chemical shift data to $233 \mathrm{~K}$ by using emulsion and down to $253 \mathrm{~K}$ bundles of thin capillaries. Their data agree after suitable susceptibility corrections with Hindman's results to $\pm 0.05 \mathrm{ppm}$ and show an increase of $\partial \delta / \partial / T$ with falling temperature. Also the pressure dependent data by Linowski et al. [15], collected between $273 \leq T \leq 373 \mathrm{~K}$, agree with their ambient pressure values after rescaling with Hindman's and Angell's result. The pressure dependence is in the range of temperature overlap identical to our data.

Our data, taken with internal standard in bulk water (Table 2), extend at $200 \mathrm{MPa}$ to $222 \mathrm{~K}$. The emulsions could be studied close to the homogeneous nucleation temperature curve, which drops from $233 \mathrm{~K}$ at $0.1 \mathrm{MPa}$ to $182 \mathrm{~K}$ at $200 \mathrm{MPa}$. Comparison of the two uncorrected data sets shows that the chemical shifts for the emulsion data are at ambient pressure smaller by $0.11 \mathrm{ppm}$, this difference increasing to $0.18 \mathrm{ppm}$ at $200 \mathrm{MPa}$. The variation of this difference with temperature is within experimental error, assuming that the difference is caused by susceptibility changes in the emulsions.

The emulsion data in Fig. 3 are thus corrected by $0.18 \mathrm{ppm}$ for the $200 \mathrm{MPa}$ isobar and $0.12 \mathrm{ppm}$ for the $20 \mathrm{MPa}$ isobar.

In Fig. 4 some isotherms for $\delta$ are given. For $T \geq 300 \mathrm{~K}$ these isotherms show the increase of $\delta$ with pressure ob- 
served for methanol over the whole $T, p$ range studied. For $T \leq 273 \mathrm{~K}$ the chemical shift decreases with pressure. This is to be taken as a weakening or breaking of the hydrogen bonded structure. However the effect remains rather small down to the lowest isotherm $(233 \mathrm{~K})$ that can be obtained.

The slope of the isobars of $\delta\left(\mathrm{H}_{2} \mathrm{O}\right)$ in Fig. 3 seems to increase at both pressures with falling temperature, contrary to the effect observed for methanol (Figure 2).

It is customary to derive from spectroscopic data on bulk water the percentage of hydrogen bonds formed [18]. Estimates from IR data yield at $273 \mathrm{~K}$ about $10 \%$ of free hydroxyl groups for water and less than $2 \%$ for methanol. The chemical shift studies do not corroborate this conclusion. For both compounds the chemical shifts increase down to the lowest temperatures studied. All ice modifications and crystalline methanol should be fully hydrogen bonded. Thus knowledge of the chemical shift of the $\mathrm{OH}$-protons in the solid phases would permit an estimate of the hydrogen bond concentration. For ice $I_{\mathrm{h}}, \delta\left(\mathrm{H}_{2} \mathrm{O}\right.$ Ice $\left.I_{\mathrm{h}}\right)$ was determined by Rhim et al. [21] at liquid nitrogen temperature to $7.9 \mathrm{ppm}$ on the TPS scale. Older data $[19,20]$ show deviations of $\pm 2 \mathrm{ppm}$, but the result quoted is considered the most reliable [22]. Accepting $\delta=7.9 \mathrm{ppm}$ as the chemical shift for an ideal hydrogen bond, leads to the conclusion that even at $183 \mathrm{~K}$ about $30 \%$ of the bonds

[1] G. A. Jeffrey, An Introduction to Hydrogen Bonding, Oxford University Press, Oxford 1997.

[2] S. Scheiner, Hydrogen Bonding, A Theoretical Perspective, Oxford University Press, Oxford 1997.

[3] A. L. Van Geet, Anal. Chem. 42, 679 (1970).

[4] R. C. Dougherty, J. Chem. Phys. 109, 7372 (1998).

[5] M. M. Hoffmann and M. S. Conradi, J. Phys. Chem. B 102, 263 (1998).

[6] M. M. Hoffmann and M. S. Conradi, J. Am. Chem. Soc. 119, 3811 (1997).

[7] N. Matubayasi, C. Wakai, and M. Nakahara, Phys. Rev. Lett. 78, 573 (1997).

[8] O. Mishima and H. E. Stanley, Nature, London, 396, 324 (1998).

[9] H. Yamada, Rev. Sci. Instrum. 45, 640 (1974).

[10] W. E. Price and H.-D. Lüdemann in: High Pressure Techniques in Chemistry and Physics: A Practical Approach, W. B. Holzapfel and N. S. Isaacs (eds.), Oxford University Press, Oxford 1997, Chapt. 5, p. 225.

[11] F. X. Prielmeier, E. W. Lang, R. J. Speedy, and H.-D. Lüdemann, Ber. Bunsenges. Phys. Chem. 92, 1111 (1988). are broken, while at $273 \mathrm{~K} 40 \%$ "free" hydroxyls are found in water. In our opinion these results, taken together with the data on methanol, show that two state models for the description of the hydrogen bonding in these two liquids are inadequate for the description of the $T, p$ dependence of the $\mathrm{H}$-bond equilibria in these fluids. Rather one has to consider that these liquids are to be described by a wide continuous distribution of hydrogen bonded states which changes with the variation of $T$ and $p$ [4]. The rather weak $p$-dependence of $\delta\left(\mathrm{H}_{2} \mathrm{O}\right)$ at the lowest isotherms $(243 \mathrm{~K}$ and $233 \mathrm{~K})$ does in our opinion not support the claim of a second critical point connected with the phase separation of a low density liquid water and a high density liquid water at $\approx 220 \mathrm{~K}$ and $100 \mathrm{MPa}$ [8]. At least this result permits only small differences between the hydrogen bond networks possible in the two liquids.

\section{Acknowledgements}

Liuping Chen thanks the China Scholarship Council for financial support. It is gratefully acknowledged that financial support was obtained from the Deutsche Forschungsgemeinschaft and the Fonds der Chemischen Industrie. It is a pleasure to thank G. Niesner and J. Gamez-Weingärtner from our faculty workshop for their fast and competent support of our work.

[12] E. M. Schulman, D. W. Dwyer, and D. C. Doetschman, J. Phys. Chem. 94, 7308 (1990).

[13] S. L. Wallen, B. J. Palmer, B. C. Garrett, and C. R. Yonker, J. Phys. Chem. 100, 3959 (1996).

[14] R. B. Martin, Chem. Rev. 96, 3043 (1996).

[15] I. W. Linowski, Nan-Liu, and J. Jonas, J. Chem. Phys. 65, 3383 (1976).

[16] J. C. Hindman, J. Chem. Phys. 44, 4582 (1966).

[17] C. A. Angell, J. Schuppert, and J. C. Tucker, J. Phys. Chem. 77, 3092 (1973).

[18] W. A. P. Luck, The Hydrogen Bond, P. Schuster, G. Zundel, and C. Sandorfy eds., North-Holland Publ., Amsterdam 1976, Vol. III, p. 1367.

[19] A. Pines, D. J. Rubens, S. Vega, and M. Mehring, Phys. Rev. Lett. 36, 110 (1976).

[20] L. M. Ryan, R. C. Wilson, and B. C. Bernstein, Chem. Phys. Lett. 52, 341 (1977).

[21] W. K. Rhim, D. P. Burum, and D. D. Elleman, J. Chem. Phys. 71, 3139 (1979).

[22] E. Brunner and U. Sternberg, Prog. Nucl. Magn. Res. Spect. 32, 21 (1998). 